\title{
Transmembrane epitope delivery by passive protein threading through the pores of the OmpF porin trimer
}

\author{
Sejeong Lee ${ }^{1}$, Nicholas G. Housden², Sandra A. lonescu ${ }^{1}$, Matthew H. Zimmer ${ }^{1}{ }^{13}$, Renata \\ Kaminska², Colin Kleanthous², Hagan Bayley ${ }^{1 *}$ \\ ${ }^{1}$ Chemistry Research Laboratory, University of Oxford, Oxford, OX1 3TA, UK \\ 2Department of Biochemistry, University of Oxford, Oxford, OX1 3QU, UK \\ ${ }^{3}$ current address: Arthur Amos Noyes Laboratory of Chemical Physics, California Institute of \\ Technology, Pasadena, CA 91125, US \\ *Corresponding author: Hagan Bayley \\ Email: hagan.bayley@chem.ox.ac.uk
}

\section{Supplementary Information}

\section{Table of Contents}

Figure S1. Pre-equilibrium fluorescence measurements for the $\mathrm{OmpF}_{-} \mathrm{OBS}_{1}$ complex formation and dissociation at different salt concentrations and $\mathrm{pH}$ values.

Figure S2. Concentration dependence of $\mathrm{OBS}_{12-18}$ peptide binding to the periplasmic side of OmpF.

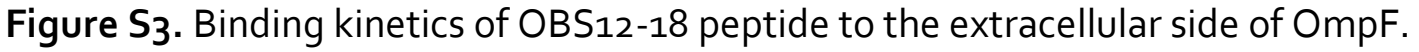

Figure $S_{4}$. Binding kinetics of the OBS254-63 peptide to the periplasmic side of OmpF.

Figure $\mathrm{S}_{5}$. OBS 1 enters $\mathrm{OmpF}$ from the periplasmic side with its $\mathrm{N}$ terminus first.

Figure S6. OBS2 does not bind in an orientation in which the $\mathrm{N}$ terminus points towards the extracellular side.

Figure S7. Current-voltage plots of individual OmpF pores before and after the addition of the IUTD and the IUTD-fusion.

Figure S8. The third OmpF subunit is occluded by the addition of OBS12-26 peptide.

Figure S9. A fusion tag also occludes the third OmpF subunit.

Figure S10. The deletion of either $\mathrm{OBS}_{1}$ or $\mathrm{OBS}_{2}$ perturbs the two-step translocation through OmpF.

Figure S11. Dependence of the translocation of the IUTD-fusion through OmpF on voltage and concentration.

Figure S12. Comparison of kinetic rate constants for OmpF translocation by the untagged IUTD and the IUTD-fusion.

Table S1. Rate constants for the interactions of the IUTD-fusion and the IUTD with OmpF.

Table S2. Rate constants for the interactions of the IUTD-fusion mutants to OmpF.

Table S3. Effective molarities of the IUTD-fusion and the IUTD-fusion mutants. 

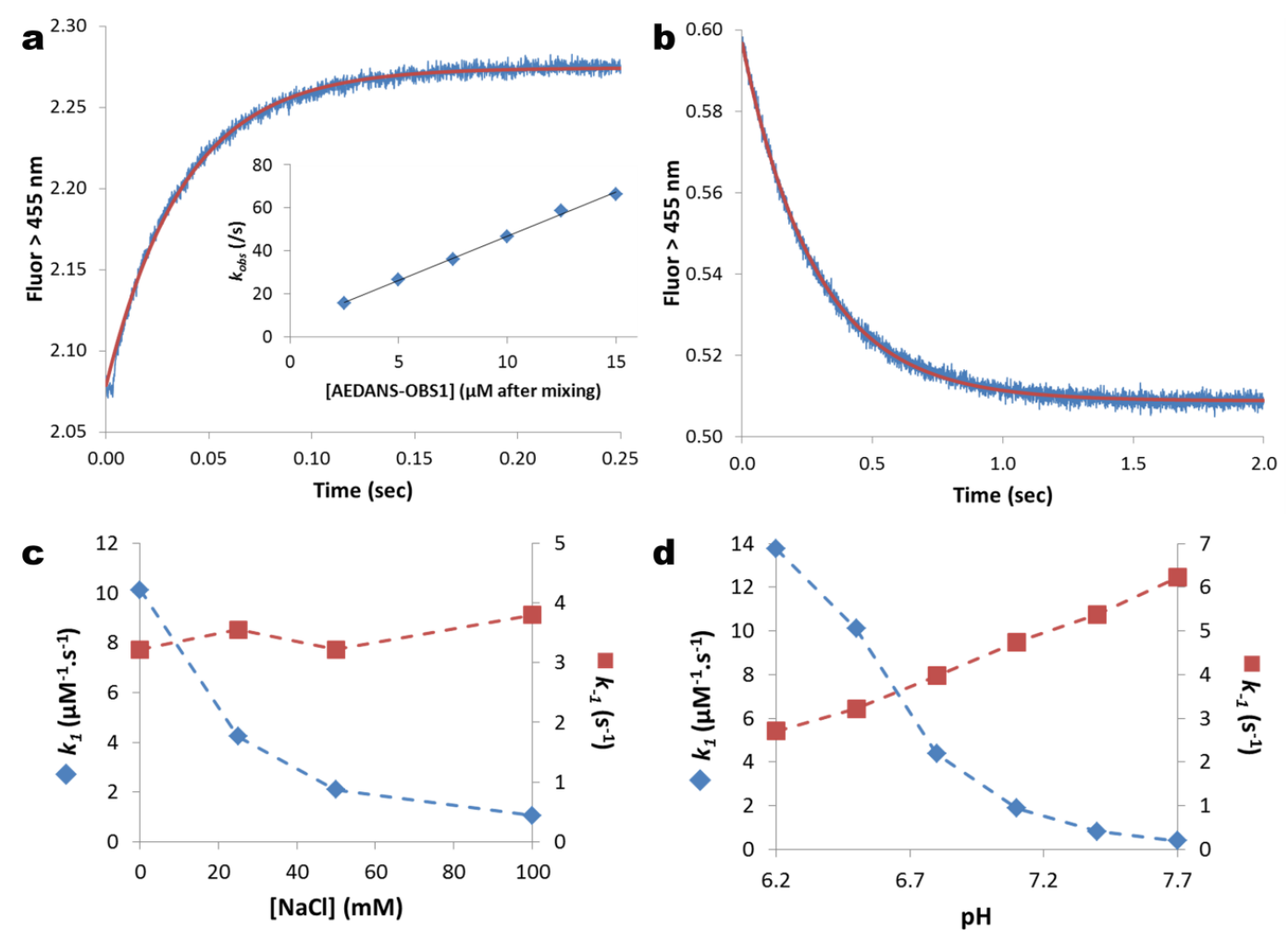

Figure S1. Pre-equilibrium fluorescence measurements for the OmpF-OBS1 complex formation and dissociation at different salt concentrations and $\mathrm{pH}$ values. $\mathrm{OBS}_{1}$ in the context of $\mathrm{OBS}_{1-32}$-E9 DNase.Img $\left(\mathrm{T}_{25} \mathrm{C}\right)$ was labelled on the cysteine with IAEDANS (OBS1-AEDANS), allowing FRET between the tryptophans of OmpF (residues 61 and 214) and the AEDANS upon complex formation. Data were collected on an Applied Photophysics SX18 stopped-flow apparatus using an excitation wavelength of $280 \mathrm{~nm}$ and fluorescence emissions $>455 \mathrm{~nm}$ selected with a cut-off filter. a, Rise in AEDANS fluorescence upon $5 \mu \mathrm{M}$ AEDANS-OBS 1 binding to $0.165 \mu \mathrm{M}$ OmpF in $20 \mathrm{mM}$ potassium phosphate, $\mathrm{pH} 6.5,25 \mathrm{mM} \mathrm{NaCl}$ and $1 \%(\mathrm{w} / \mathrm{v}) \beta-\mathrm{OG}$, at $10^{\circ} \mathrm{C}$. Data were fitted to a single exponential equation to determine $k_{o b s}$, and $k_{1}$ was determined from the gradient of $k_{o b s}$ plotted against AEDANS-OBS 1 concentration $\left(k_{1}=9.80 \pm 0.31 \mu \mathrm{M}^{-1} \mathrm{~s}^{-1}\right)$. b, Dissociation of the complex formed between $0.33 \mu \mathrm{M}$ OmpF and $1 \mu \mathrm{M}$ AEDANS-OBS 1 after mixing with 20 $\mu \mathrm{M}$ unlabelled $\mathrm{OBS}_{1}$ peptide. The dissociation rate constant $\left(k_{-1}=3.22 \pm 0.03 \mathrm{~s}^{-1}\right)$ was determined by fitting the data to a single exponential equation. The errors are determined from the deviation of two repeats. c, Effect of ionic strength on AEDANS-OBS1 binding OmpF. Association and dissociation rate constants were measured in $20 \mathrm{mM}$ potassium phosphate, $\mathrm{pH} 6.5,1 \%(\mathrm{w} / \mathrm{v}) \beta-\mathrm{OG}$, varying $\mathrm{NaCl}$ concentration between o and $100 \mathrm{mM}$. The association rate constant decreased with increasing $\mathrm{NaCl}$ concentration, whereas the dissociation rate constant remained the same. In comparison with no salt, $100 \mathrm{mM} \mathrm{NaCl}$ resulted in a 10-times lower affinity of $\mathrm{OBS}_{1}$ binding to OmpF. $\mathbf{d}_{1}$ Impact of $\mathrm{pH}$ on $\mathrm{AEDANS}_{\mathrm{O}} \mathrm{OBS}_{1}$ binding to OmpF. Association and dissociation rate constants were measured in $20 \mathrm{mM}$ potassium phosphate, $1 \%(\mathrm{w} / \mathrm{v}) \beta$-OG over the $\mathrm{pH}$ range of 6.2 to 7.7 . 
The association rate constant decreased with increasing $\mathrm{pH}$, by up to 80 -fold, whereas the dissociation rate constant increased only by a factor of 2 . 
a
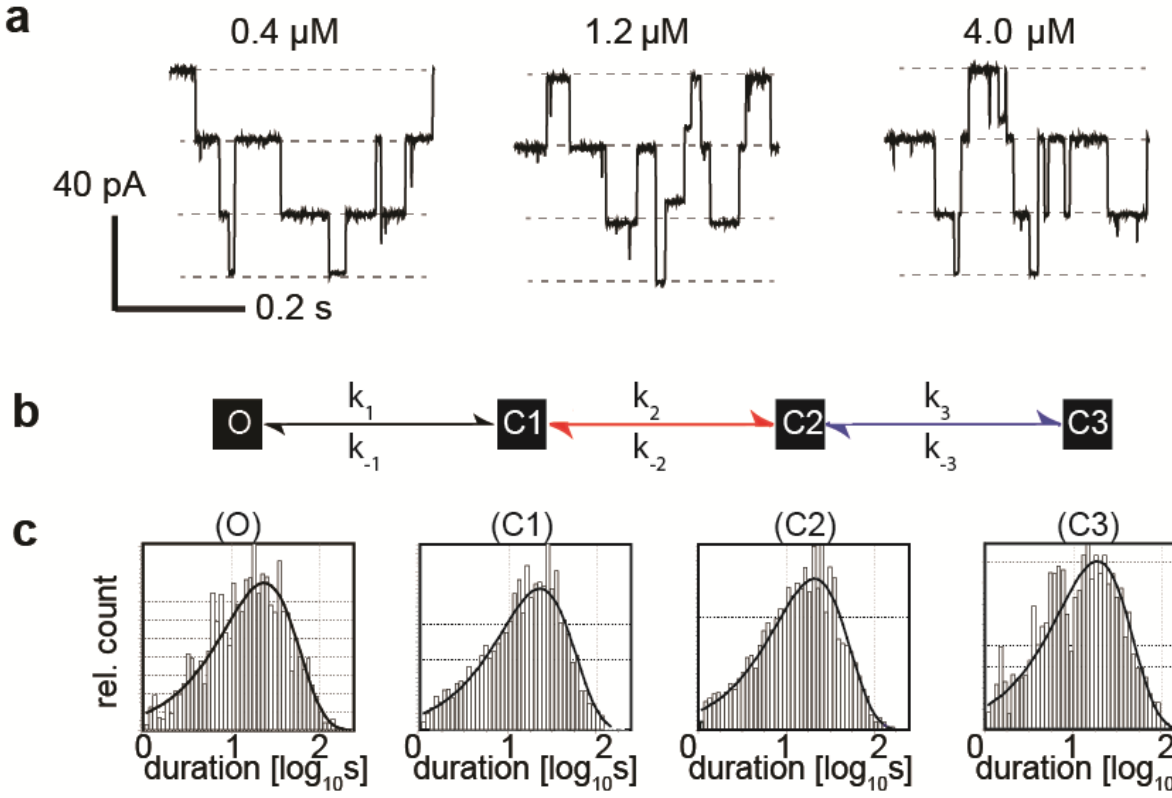

d
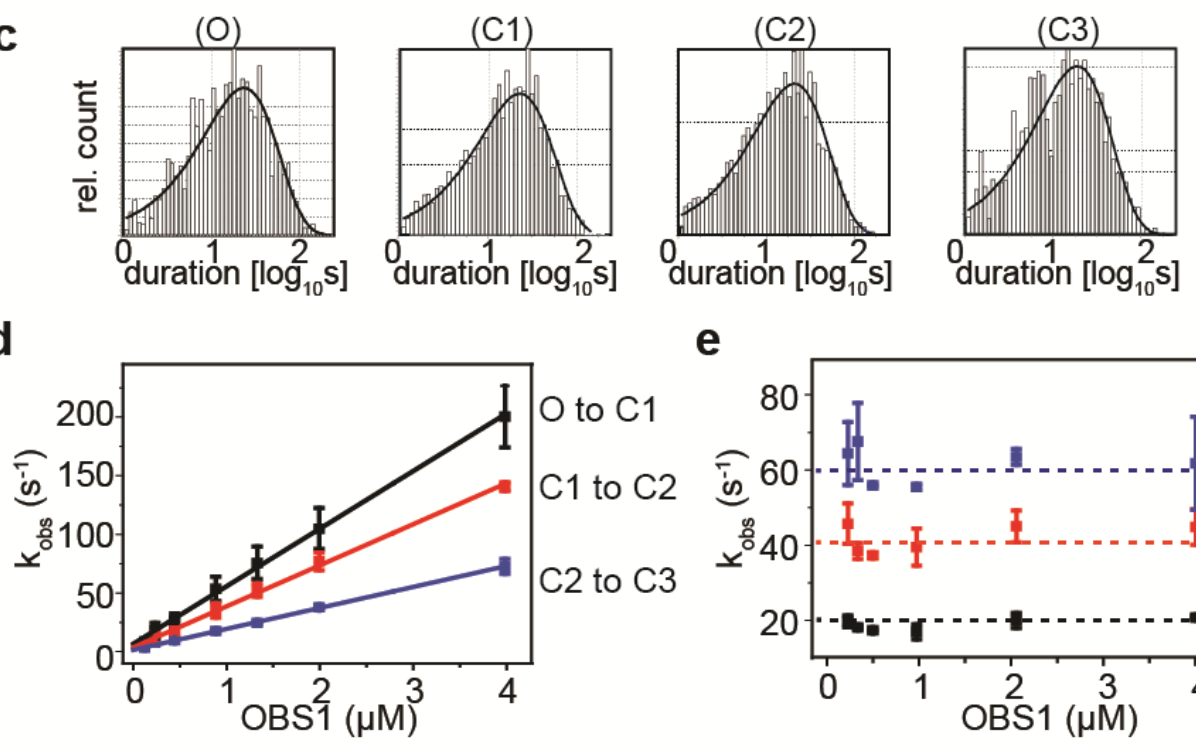

e

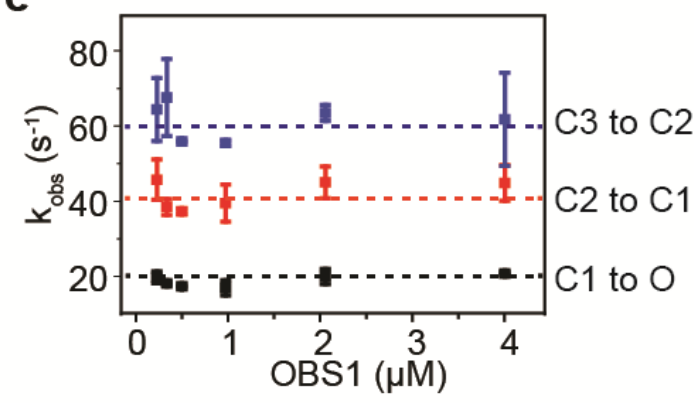

Figure S2. Concentration dependence of $\mathrm{OBS}_{1_{2-18}}$ peptide binding to the periplasmic side of OmpF. a, Current traces of OmpF after $0.4 \mu \mathrm{M}, 1.2 \mu \mathrm{M}$, and $4.0 \mu \mathrm{M} \mathrm{OBS} 1_{2-18}$ were added to the periplasmic side of $\mathrm{OmpF}$ at an applied potential of $+100 \mathrm{mV}$ in phosphate buffer $\mathrm{pH} 6.4,100 \mathrm{mM} \mathrm{KCl}$. First, second, and third subunit occlusions were observed in a stepwise manner. $\mathbf{b}, \mathrm{A}$ reaction scheme describing the sequential occlusion of OmpF. $\mathbf{c}$, Normalized histograms of dwell-times in each state of OmpF in presence of $0.4 \mu \mathrm{M} O B S_{1}$ peptide on the periplasmic side. The data included thousands of binding events. Mean dwell-times and $k_{\text {obs }}$ values (for both association and dissociation) were obtained by fitting the histograms to maximum interval likelihood functions by using QuB software. $d$, Concentration dependence of $\mathrm{OBS}_{1_{2-18}}$ association with $\mathrm{OmpF}$. Association rate constants $\left(k_{1}, k_{2}, k_{3}\right)$ were determined from the linear dependence of $k_{\text {obs }}$ values on $\mathrm{OBS}_{1-18}$ concentration. e, Concentration independent dissociation of $\mathrm{OBS}_{1_{2-18}}$ from $\mathrm{OmpF}$. Dissociation rate constants $\left(\mathrm{k}_{-1}, \mathrm{k}_{-2}, \mathrm{k}_{-3}\right)$ were determined by taking average values of $\mathrm{k}_{\text {obs }}$ over the range of $\mathrm{OBS}_{1_{2-18}}$ concentrations. At least three repeats at each concentration were performed. 

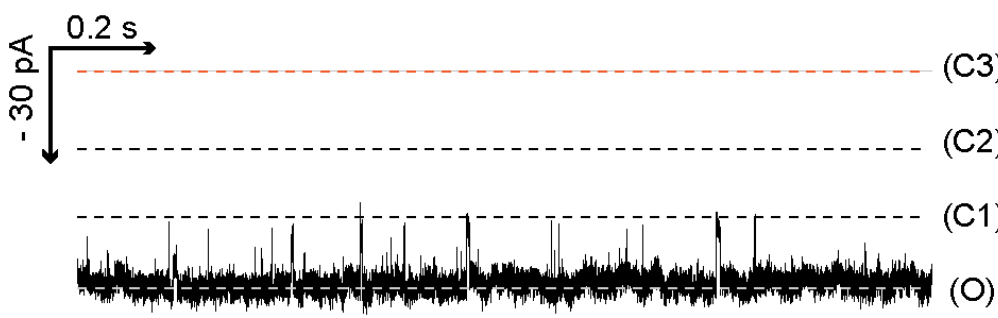

b
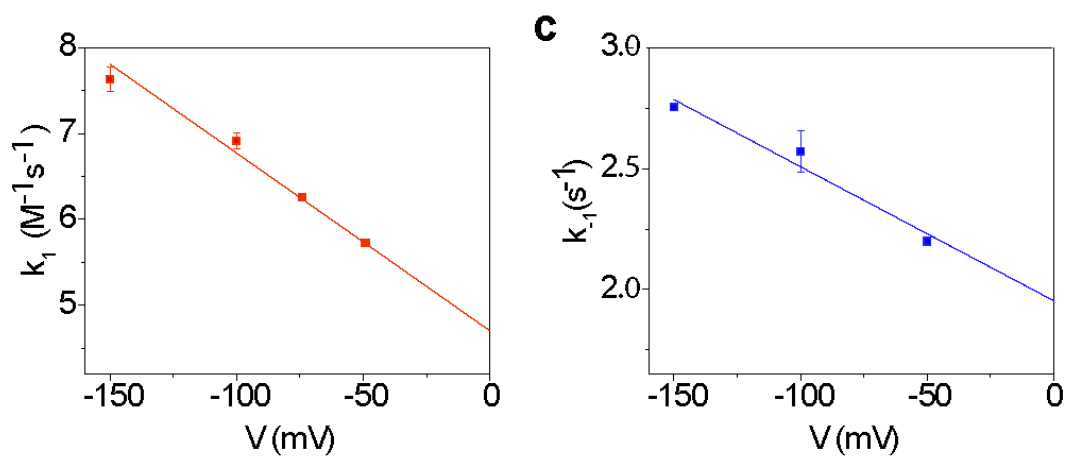

d

\begin{tabular}{|ll|c|}
\hline $\mathrm{k}_{1}\left(10^{6} \mathrm{M}^{-1} \mathrm{~s}^{-1}\right)$ & $0.050 \pm 0.013$ \\
\hline $\mathrm{k}_{-1}\left(\mathrm{~s}^{-1}\right)$ & $90 \pm 25$ \\
\hline $\mathrm{K}_{\mathrm{d}}(\mathrm{mM})$ & $5.4 \pm 2.1$ \\
\hline
\end{tabular}

Figure $S_{3}$. Binding kinetics of $\mathrm{OBS}_{1-18}$ peptide to the extracellular side of OmpF. $\mathrm{a}_{1} \mathrm{~A}$ representative current trace of $\mathrm{OBS}_{2-18}(8 \mu \mathrm{M})$ binding to the extracellular side of $\mathrm{OmpF}$ at $-100 \mathrm{mV}$ in phosphate buffer $\mathrm{pH} 6.4,100 \mathrm{mM} \mathrm{KCl}$. Reversible one-step binding events of $\mathrm{OBS}_{1_{2-18}}$ to OmpF were observed. $\mathbf{b}, A$ plot of the logarithm of the association rate constant of $\mathrm{OBS}_{1_{2-18}}$ to $\mathrm{OmpF}$ from the extracellular side $\left(\mathrm{k}_{1}\right)$ versus the applied potential. $\mathbf{c}_{\text {, }}$ A plot of the logarithm of the dissociation rate constant of $\mathrm{OBS}_{2-18}$ from the extracellular side $\mathrm{OmpF}\left(\mathrm{k}_{-1}\right)$ versus the applied potential. $\mathbf{d}$, Table of rate constants at o $\mathrm{mV}$ estimated by extrapolating the linear dependences shown in (b) and (c). The dissociation constant $\left(\mathrm{K}_{\mathrm{d}}\right)$ for the monomer was determined, considering the stochastic effect associated with the OmpF trimer, by dividing $\mathrm{k}_{-1}$ by one-third of $\mathrm{k}_{1}$. The data included thousands of binding events. At least two repeats were performed at each concentration and applied potential. Errors are the standard deviations of the repeats. 
a

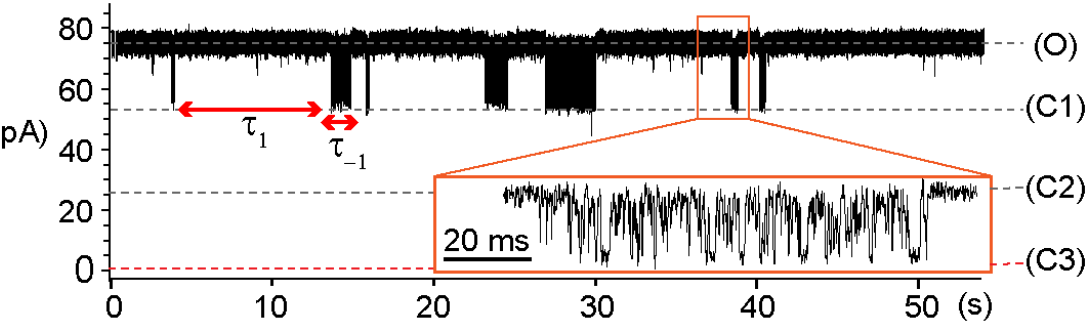

b
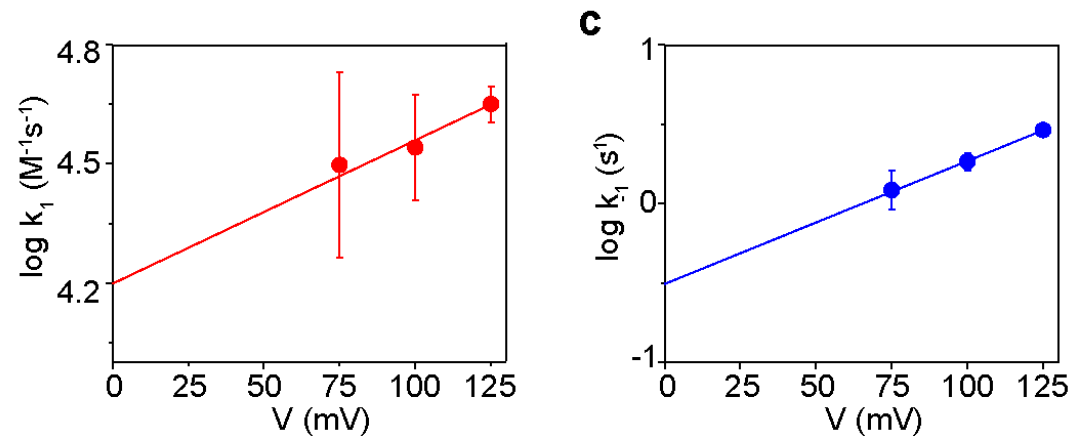

d

\begin{tabular}{|l|c|}
\hline$k_{1}\left(10^{6} M^{-1} s^{-1}\right)$ & $0.015 \pm 0.009$ \\
\hline$k_{-1}\left(s^{-1}\right)$ & $0.3 \pm 0.1$ \\
\hline$K_{d}(\mu M)$ & $60 \pm 39$ \\
\hline
\end{tabular}

Figure $\mathrm{S}_{4}$. Binding kinetics of the $\mathrm{OBS}_{2_{54-63}}$ peptide to the periplasmic side of OmpF. a, A representative current trace of $\mathrm{OBS}_{254-63}(5 \mu \mathrm{M})$ binding to the periplasmic side of OmpF $\left(+100 \mathrm{mV}\right.$ ) in phosphate buffer $\mathrm{pH} 6.4,100 \mathrm{mM} \mathrm{KCl}$. The binding of $\mathrm{OBS}_{254-63}$ to OmpF caused subsecond-to-seconds-long clusters of events as shown in the orange box. OBS2 $2_{54-}$ ${ }_{63}$ may remain associated with $\mathrm{OmpF}$ at the periplasmic entrance after initial binding and unbinding. Thus, we defined dwell times for the unoccupied state and the occupied state as $\tau_{1}$ and $\tau_{-1}$ with a cutoff of $10 \mathrm{~ms}$. Mean dwell-times were determined by using QuB software. b, A plot of the logarithm of the association rate constant of $\mathrm{OBS}_{254-63}$ to OmpF from the periplasmic side $\left(\mathrm{k}_{1}\right)$ versus the applied potential. c, A plot of the logarithm of the dissociation rate constant of $\mathrm{OBS}_{254-63}$ from the periplasmic side $\mathrm{OmpF}\left(\mathrm{k}_{-1}\right)$ versus the applied potential. $\mathbf{d}$, Table of rate constants at o $\mathrm{mV}$ estimated by extrapolating the linear dependences shown in (b) and $(c)$. The dissociation constant $\left(K_{d}\right)$ for the monomer was determined from $k_{1}$ and $k_{-1}$, by dividing $k_{1}$ by three to remove the stochastic effect associated with the OmpF trimer. The data included hundreds of binding events. At least two repeats were performed at each concentration and applied potential. Errors are the standard deviations of the repeats. 
a

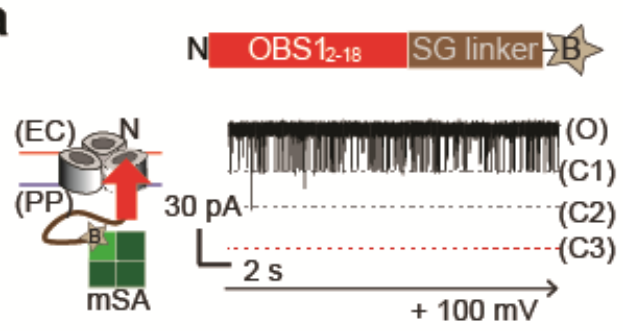

b

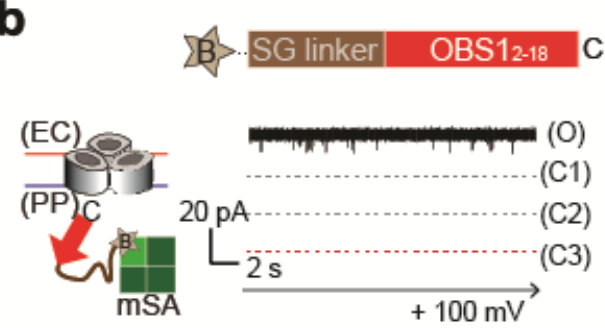

Figure $\mathrm{S}_{5}$. OBS1 enters $\mathrm{OmpF}$ from the periplasmic side with its $\mathbf{N}$ terminus first. Current traces of OmpF upon the addition of (a) the C-terminus capped or (b) the $\mathrm{N}$ terminus capped OBS1 sequence to the periplasmic side at an applied potential of $+100 \mathrm{mV}$ in phosphate buffer $\mathrm{pH} 6.4,100 \mathrm{mM} \mathrm{KCl}$. The C-terminus capped OBS1 and the $\mathrm{N}$-terminus capped $\mathrm{OBS}_{1}$ were prepared by incubating biotinylated OBS1 constructs and monovalent streptavidin (mSA) at a molar ratio of 1:1. Biotinylated $\mathrm{OBS}_{1}$ conjugates contain biotinylated cysteine (grey star) either at the C-terminus (for the C-terminus capped) or at the $\mathrm{N}$-terminus (for the $\mathrm{N}$-terminus capped), separated from the $\mathrm{OBS}_{1}$ sequence by a serine glycine (SG) linker (brown bar). a, Upon the addition of the C-terminus capped OBS1mSA (OBS1-mSA, $2 \mu \mathrm{M}$ ) one-step reversible binding events were observed. This indicates $\mathrm{OBS}_{1}$ can enter from the periplasmic entrance with its $\mathrm{N}$-terminus first. $\mathbf{b}$, Upon the

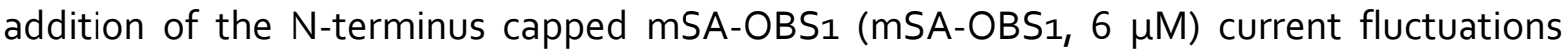
occurred only sparsely. This indicates that mSA-OBS1 does not enter OmpF with its Cterminus first. At least three independent experiments were performed in each case. 
a

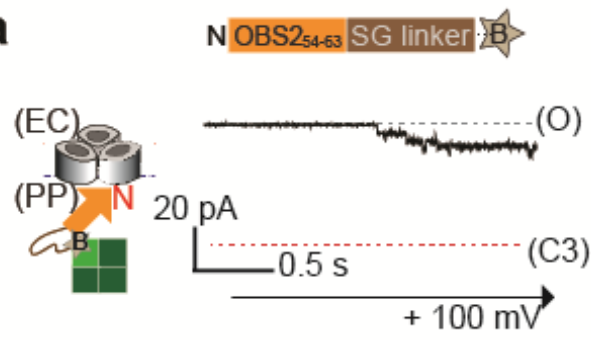

b

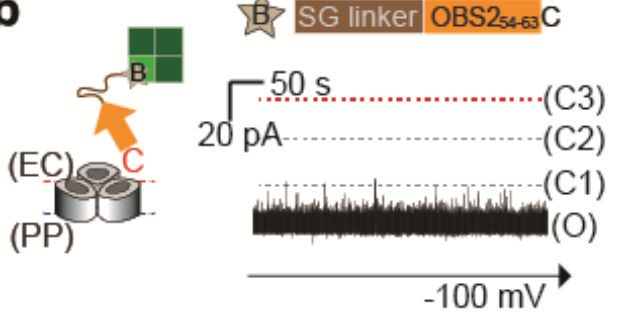

Figure S6. OBS2 does not bind in an orientation in which the $\mathrm{N}$ terminus points towards the extracellular side. Current traces of OmpF upon the addition of (a) the C-terminus capped OBS2 complex (OBS2-mSA) to the extracellular side and (b) the C-terminus capped $\mathrm{OBS}_{2}$ complex (mSA-OBS2) to the periplasmic side in phosphate buffer $\mathrm{pH} 6.4,100 \mathrm{mM}$ $\mathrm{KCl}$. The OBS2-mSA and mSA-OBS2 complexes were prepared using the same protocol as described previously (Fig. S5). a, The addition of the C-terminus capped OBS2-mSA complex $(1 \mu \mathrm{M})$ to the periplasmic side at applied potential of $+100 \mathrm{mV}$ produced minor current changes, up to $10 \mathrm{pA}$, which is less than a half the conductance of a single OmpF subunit. Possibly, OBS2 associates with the periplasmic mouth of OmpF but cannot enter the lumen with its $\mathrm{N}$ terminus first. $\mathbf{b}$, The addition of the $\mathrm{N}$-terminus capped mSA-OBS2 complex $(5 \mu \mathrm{M})$ to the extracellular side showed hardly any current fluctuations over 10 minutes, indicating that OBS2 does not enter OmpF from the extracellular side with its $C$ terminus first. At least three independent experiments were performed in each case. 
a

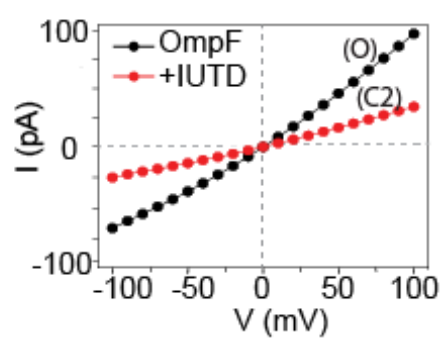

b

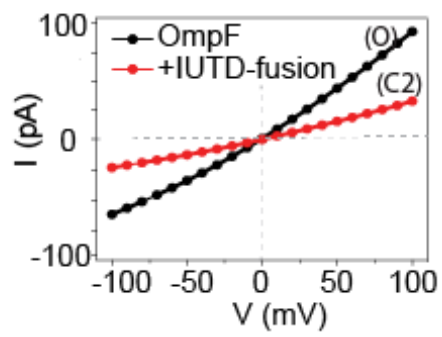

Figure S7. Current-voltage plots of individual OmpF pores before and after the addition of the IUTD and the IUTD-fusion. OmpF shows a positive asymmetric I-V curve with higher conductance at higher potential (cis-extracellular, trans-periplasmic, black). After the addition to the extracellular side of $\mathbf{a}$, untagged IUTD or $\mathbf{b}$, the IUTD-fusion, the conductance of OmpF decreased to one-third ( $\mathrm{C}_{2}$ state) at both positive and negative applied potentials (red) and the I-V curve remained positive asymmetric. 


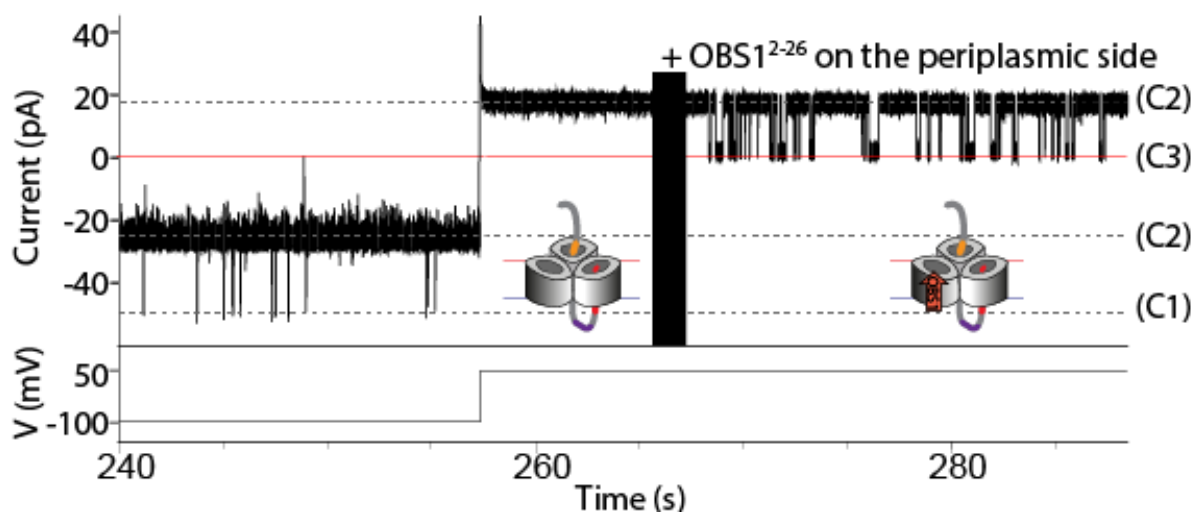

Figure S8. The third OmpF subunit is occluded by the addition of $\mathrm{OBS}_{\mathbf{1}_{\mathbf{2}-2} 6}$ peptide. The availability of the third OmpF pore was examined after blockade by untagged IUTD followed by the addition of the $\mathrm{OBS}_{1_{2-2} 6}$ peptide to the periplasmic side. Upon the addition of untagged IUTD to the extracellular side, two OmpF subunits were occluded $\left(C_{2}\right)$ at an applied potential of $-100 \mathrm{mV}$. To avoid additional events, the remaining IUTD on the extracellular side was removed by repeated buffer exchange. At $+50 \mathrm{mV}, 1 \mu \mathrm{M} O \mathrm{OBS}_{12-26}$ peptide was added to the periplasmic side and reversible binding events were observed between current levels $C_{2}$ and $C_{3}$. This indicates that the third subunit is available to bind peptide. 
a

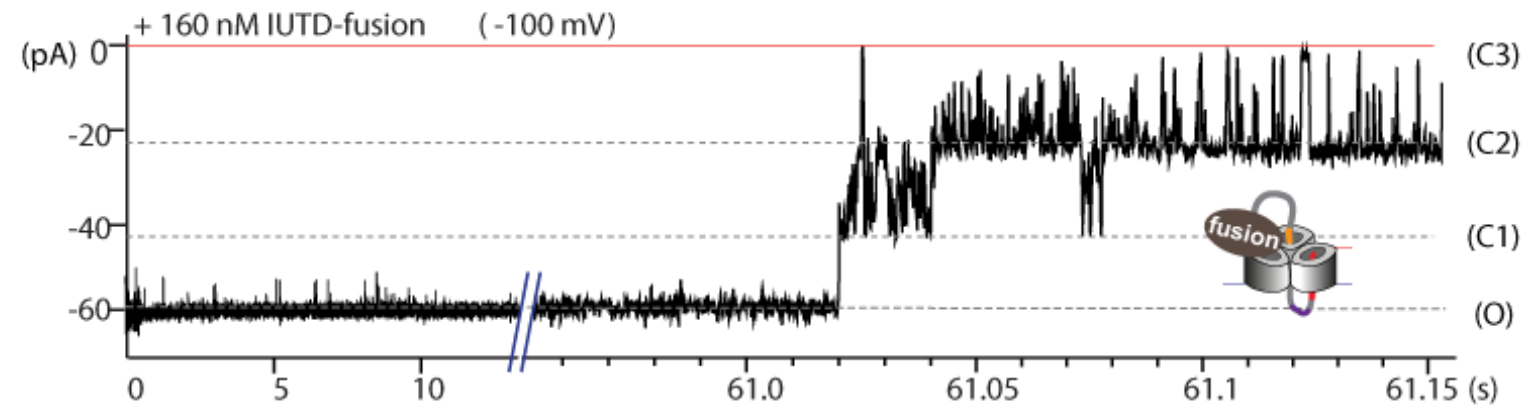

b

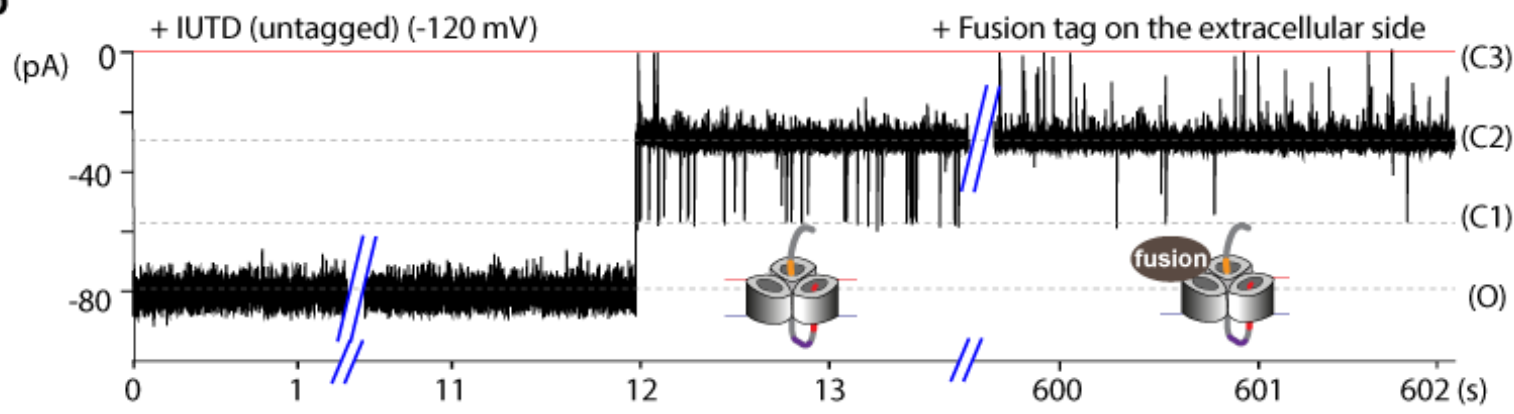

Figure S9. A fusion tag also occludes the third OmpF subunit. a, A current trace of OmpF after the addition of the IUTD-fusion construct $(160 \mathrm{nM})$ to the extracellular side at an applied potential of $-100 \mathrm{mV}$ in phosphate buffer $\mathrm{pH} 6.4,100 \mathrm{mM} \mathrm{KCl}$. As previously observed with untagged IUTD (Fig. 3b), the conductance was reduced to one-third of the open level ( $\mathrm{C}_{2}$ ) and remained there for most of time. However, the IUTD-fusion construct produced additional fluctuations between levels $C_{2}$ and $C_{3} . b_{1} A$ current trace of OmpF upon the addition of $100 \mathrm{nM}$ untagged IUTD followed by $2 \mu \mathrm{M}$ fusion-tag only to the extracellular side at an applied potential of $-120 \mathrm{mV}$. After the addition of the fusion-tag, fluctuations between levels $C_{2}$ and $C_{3}$ appeared. These data suggest that the transitions between $\mathrm{C}_{2}$ and $\mathrm{C}_{3}$ are produced by the binding of the fusion tag to the extracellular surface of OmpF. 
a

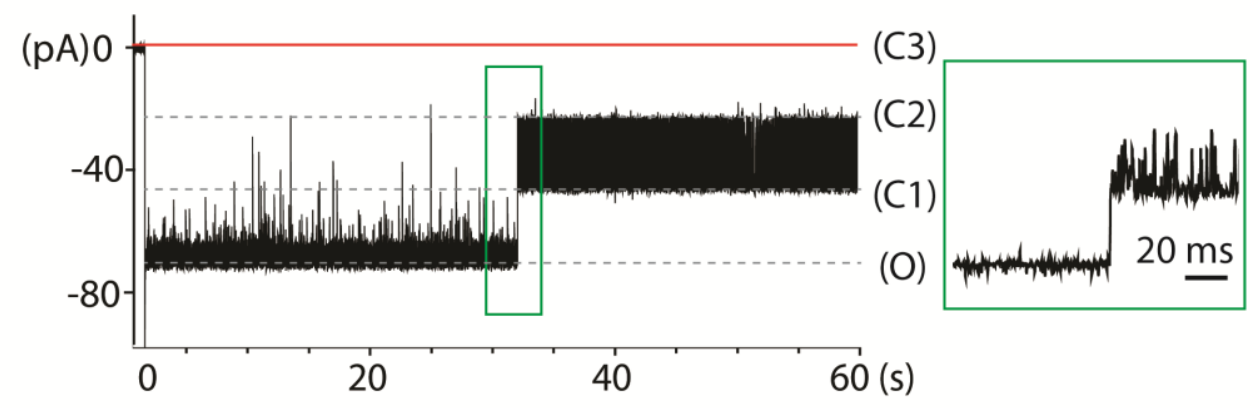

b

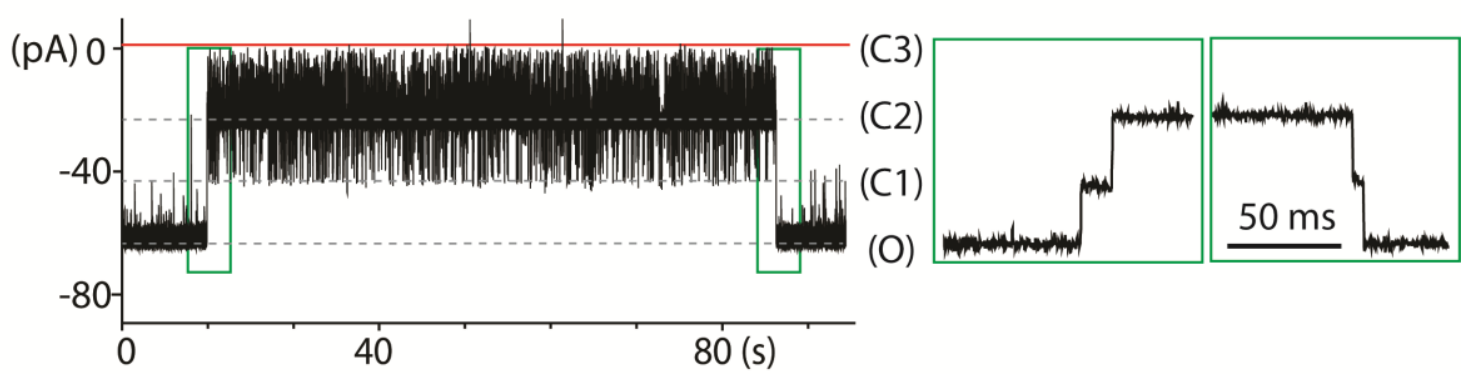

Figure S10. The deletion of either OBS1 or OBS2 perturbs the two-step translocation through OmpF. a, A current trace of OmpF with $1 \mu \mathrm{M}$ IUTD( $\triangle \mathrm{OBS} 1)$-fusion, lacking 32 residues at the $\mathrm{N}$ terminus, at an applied potential of $-100 \mathrm{mV}$ in phosphate buffer $\mathrm{pH}$ 6.4, $100 \mathrm{mM} \mathrm{KCl}$. The green box shows a zoomed-in region. Without the OBS1 sequence, only transient binding to the second-subunit was observed. $\mathbf{b}, \mathrm{A}$ current trace of OmpF with 1 $\mu \mathrm{M}$ IUTD( $\triangle \mathrm{OBS} 2$ )-fusion, lacking the $\mathrm{OBS}_{254-63}$ sequence, under the same conditions as in S10a. The green boxes show zoomed-in regions. The two-step translocation was observed but it was reversed within $1 \mathrm{~min}$. The third subunit occlusion $\left(\mathrm{C}_{3}\right)$ is induced by the fusiontag. At least three independent experiments were performed in each case. 
a

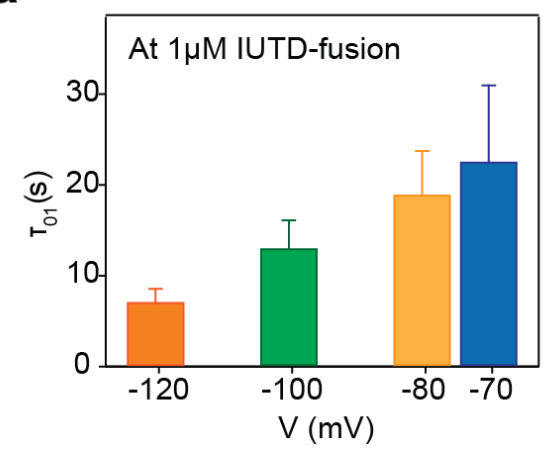

C

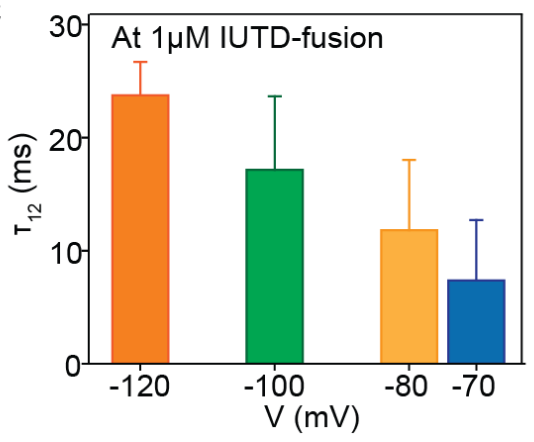

b

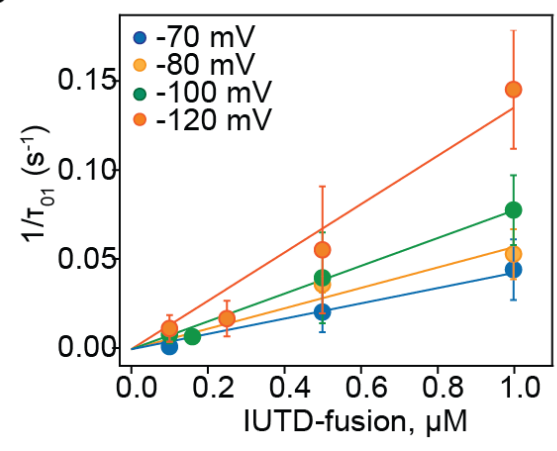

d

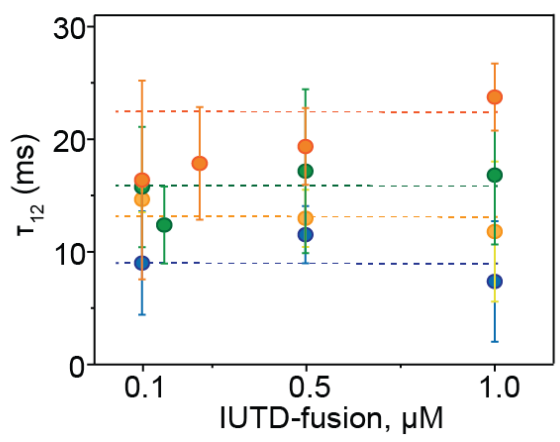

Figure S11. Dependence of the translocation of the IUTD-fusion through OmpF on voltage and concentration. a, A bar chart of mean dwell times before the association of 1 $\mu M$ IUTD-fusion with OmpF $\left(\tau_{01}\right)$ at different applied potentials. The values for the mean dwell times ( \pm standard deviation) are $30 \pm 6.7 \mathrm{~s}$ at $-70 \mathrm{mV}, 24 \pm 6.1 \mathrm{~s}$ at $-80 \mathrm{mV}, 15 \pm 2.9 \mathrm{~s}$ at $-100 \mathrm{mV}$, and $11 \pm 4 \mathrm{~s}$ at $-120 \mathrm{mV}$. $\mathbf{b}$, The concentration dependence of IUTD-fusion binding to the extracellular side $O \mathrm{mpF}$, plotting the inverse of the mean dwell times $\left(1 / \tau_{01}\right)$. The association rate constants $\left(\mathrm{k}_{\mathrm{O}_{1}}\right)$ at various potentials were determined by fitting the linear

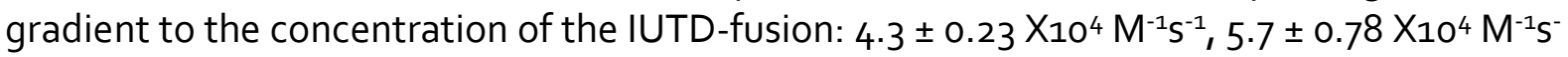

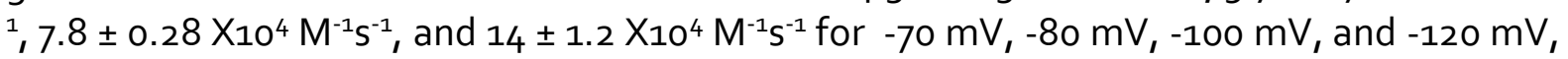
respectively. $\mathbf{c}, \mathrm{A}$ bar chart of mean dwell times ( \pm standard deviation) for the second step in the interaction of $1 \mu \mathrm{M}$ IUTD-fusion with $\mathrm{OmpF}\left(\tau_{12}\right)$ at different applied potentials. Mean dwell-times are: $7.4 \pm 5.4 \mathrm{~ms}$ at $-70 \mathrm{mV}, 12 \pm 6.2 \mathrm{~ms}$ at $-80 \mathrm{mV}, 17 \pm 6.5 \mathrm{~ms}$ at $-100 \mathrm{mV}$, and $24 \pm 3.0 \mathrm{~ms}$ at $-120 \mathrm{mV}$, respectively. $d$, The concentration independence of the second step. Dashed lines indicate the averages of the mean dwell-times over the various concentrations. The association rate constants $(\mathrm{k} 12)$ at various potentials were determined by taking the average of $1 / \tau_{12}$. 
a

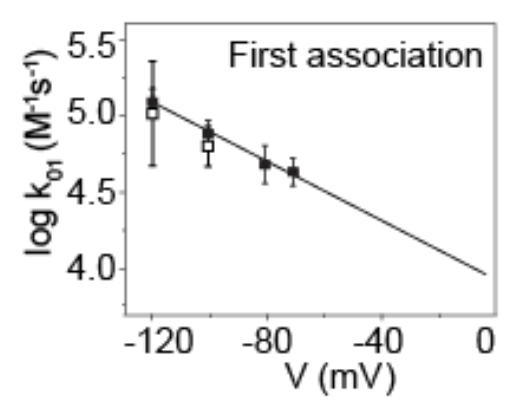

b

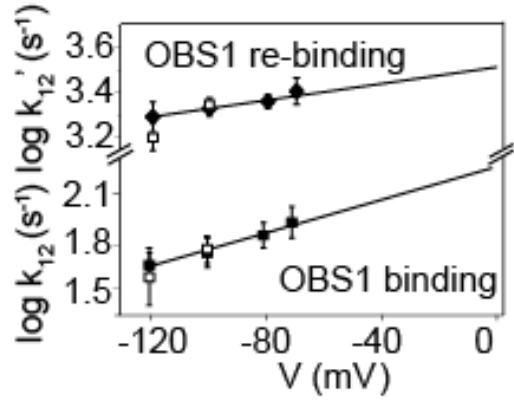

C

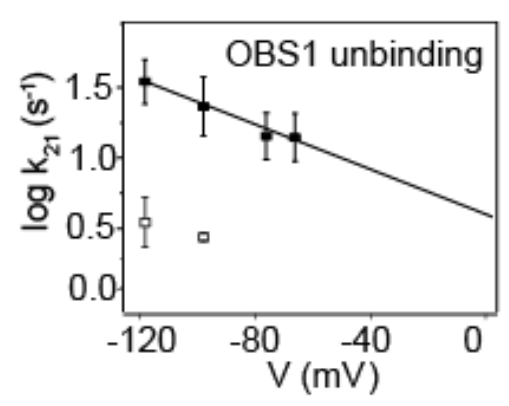

Figure S12. Comparison of kinetic rate constants for OmpF translocation by the untagged IUTD and the IUTD-fusion. a, A plot of the logarithm of association rate constants $\left(\mathrm{k}_{01}\right)$ versus the applied potential: untagged IUTD ( $(\mathrm{)})$; IUTD-fusion $(\bullet)$. $\mathbf{b}$, A plot of the logarithm of rate constants for binding to the second subunit (initial binding $k_{12}$ rebinding $\mathrm{k}_{12}{ }^{\prime}$ ) versus the applied potential. $c_{\text {, }}$ A plot of the logarithm of rate constants for unbinding from the second subunit of $\operatorname{OmpF}\left(k_{21}\right)$ versus the applied potential. For $k_{01}, k_{12}$, and $k_{12}{ }^{\prime}$, there are no observable differences between untagged IUTD and the IUTD-fusion. For unbinding, there is an about ten-fold difference in $\mathrm{k}_{21}$, probably due to the presence of the fusion-tag. 
Table S1. Rate constants for the interactions of the IUTD-fusion and the IUTD with OmpF

\begin{tabular}{|c|c|c|c|c|}
\hline & $-70 \mathrm{mV}$ & $-80 \mathrm{mV}$ & $-100 \mathrm{mV}$ & $-120 \mathrm{mV}$ \\
\hline & \multicolumn{4}{|c|}{ Association with a first subunit: $\mathrm{k}_{01}\left(10^{4} \mathrm{M}^{-1} \mathrm{~s}^{-1}\right)$} \\
\hline IUTD-fusion & $4.5 \pm 0.33$ & $7.5 \pm 1.3$ & $9.7 \pm 1.1$ & $14 \pm 0.65$ \\
\hline \multirow[t]{2}{*}{ IUTD* } & - & - & $6.3 \pm 1.9$ & $10 \pm 4.1$ \\
\hline & \multicolumn{4}{|c|}{ Initial binding of the OBS1 sequence to a second subunit: $\mathrm{k}_{12}\left(\mathrm{~s}^{-1}\right)$} \\
\hline IUTD-fusion & $80 \pm 19$ & $68 \pm 13$ & $52 \pm 11$ & $44 \pm 8.1$ \\
\hline \multirow[t]{2}{*}{ IUTD } & - & - & $58 \pm 13$ & $38 \pm 17$ \\
\hline & \multicolumn{4}{|c|}{ Rebinding of the OBS1 sequence to a second subunit: $\mathrm{k}_{12}{ }^{\prime}\left(\mathrm{s}^{-1}\right)$} \\
\hline IUTD-fusion & $2800 \pm 410$ & $2300 \pm 190$ & $2000 \pm 200$ & $1800 \pm 330$ \\
\hline \multirow[t]{2}{*}{ IUTD } & - & - & $2200 \pm 270$ & $1400 \pm 340$ \\
\hline & \multicolumn{4}{|c|}{ Unbinding of the OBS 1 sequence from the second subunit: $k_{21}\left(s^{-1}\right)$} \\
\hline IUTD-fusion & $12 \pm 5 \cdot 2$ & $13 \pm 5 \cdot 3$ & $22 \pm 11$ & $35 \pm 13$ \\
\hline IUTD & - & - & $2.6 \pm 1.1$ & $3.4 \pm 0.7$ \\
\hline
\end{tabular}

Rate constants represent the mean value $( \pm$ s.d.) obtained from at least three and up to twenty independent experiments under the various conditions.

* To initiate translocation of the free IUTD at submicromolar concentrations, we applied higher potentials, $-100 \mathrm{mV}$ and $-120 \mathrm{mV}$. 
Table S2. Rate constants for the interactions of the IUTD-fusion mutants to OmpF

\begin{tabular}{|c|c|c|c|c|}
\hline & $-70 \mathrm{mV}$ & $-80 \mathrm{mV}$ & $-100 \mathrm{mV}$ & $-120 \mathrm{mV}$ \\
\hline & \multicolumn{4}{|c|}{ Association with a first subunit: $\mathrm{k}_{01}\left(10^{4} \mathrm{M}^{-1} \mathrm{~s}^{-1}\right)$} \\
\hline IUTD $(\Delta 13)$-fusion* & $9.2 \pm 2.7$ & - & $33 \pm 12$ & - \\
\hline IUTD( $(\Delta 6)$-fusion & $2.2 \pm 0.94$ & $2.4 \pm 1.1$ & $3.3 \pm 0.8$ & $4.7 \pm 3.1$ \\
\hline IUTD(12T)-fusion & $3.8 \pm 0.51$ & $6.3 \pm 1.4$ & $13 \pm 5.2$ & $30 \pm 5.1$ \\
\hline \multirow[t]{2}{*}{ IUTD(T6)-fusion } & $3.6 \pm 0.85$ & $3.5 \pm 2.2$ & $4.9 \pm 1.7$ & $7.5 \pm 2.0$ \\
\hline & \multicolumn{4}{|c|}{ Initial binding of the OBS1 sequence to a second subunit: $\mathrm{k}_{12}\left(\mathrm{~s}^{-1}\right)$} \\
\hline IUTD( $\Delta 13)$-fusion & $5.5 \pm 3.8$ & - & $3.0 \pm 0.7$ & - \\
\hline IUTD( $(\Delta 6)$-fusion & $24 \pm 4.7$ & $18 \pm 2.6$ & $17 \pm 4 \cdot 4$ & $13 \pm 8.1$ \\
\hline IUTD(12T)-fusion & $100 \pm 59$ & $98 \pm 18$ & $71 \pm 33$ & $70 \pm 9.0$ \\
\hline \multirow{2}{*}{ IUTD(T6)-fusion } & $51 \pm 9.6$ & $46 \pm 15$ & $38 \pm 13$ & $32 \pm 6.7$ \\
\hline & \multicolumn{4}{|c|}{ Rebinding of the OBS1 sequence to a second subunit: $\mathrm{k}_{12}{ }^{\prime}\left(\mathrm{s}^{-1}\right)$} \\
\hline IUTD $(\Delta 6)$-fusion & $2100 \pm 310$ & $2100 \pm 100$ & $1800 \pm 280$ & $1500 \pm 310$ \\
\hline IUTD(12T)-fusion & $1700 \pm 510$ & $1600 \pm 80$ & $1400 \pm 140$ & $1300 \pm 90$ \\
\hline \multirow[t]{2}{*}{ IUTD(T6)-fusion } & $2600 \pm 330$ & $1900 \pm 440$ & $1400 \pm 40$ & $870 \pm 70$ \\
\hline & \multicolumn{4}{|c|}{ Unbinding of the OBS 1 sequence from the second subunit: $k_{21}\left(s^{-1}\right)$} \\
\hline IUTD( $(\Delta 6)$-fusion & $16 \pm 10$ & $8.9 \pm 1.6$ & $27 \pm 13$ & $39 \pm 2.4$ \\
\hline IUTD(12T)-fusion & $0.4 \pm 0.16$ & $5.3 \pm 1.3$ & $5.3 \pm 1.9$ & $1.7- \pm 0.6$ \\
\hline IUTD(T6)-fusion & $2.6 \pm 0.3$ & $1.9 \pm 0.6$ & $5.1 \pm 1.4$ & $6.9 \pm 0.58$ \\
\hline
\end{tabular}

Rate constants are the mean value ( \pm s.d.) obtained from at least three independent experiments under the various conditions.

* The IUTD $\left(\Delta_{13}\right)$-fusion showed few transitions between $C_{1}$ and $C_{2}$. Therefore, only the rate constants for the initial associations $\left(k_{01}\right.$ and $\left.k_{12}\right)$ are given here. 
Table S3. Effective molarities of the IUTD-fusion and the IUTD-fusion mutants

\begin{tabular}{|c|c|c|}
\hline & $\mathrm{EM}^{1}(\mathrm{mM})$ & $\mathrm{EM}^{2}(\mathrm{mM})$ \\
\hline IUTD-fusion & $0.030 \pm 0.006$ & $0.91 \pm 0.26$ \\
\hline IUTD( $\Delta 6)$-fusion & $0.008 \pm 0.003$ & $0.58 \pm 0.16$ \\
\hline IUTD(12T)-fusion & $0.031 \pm 0.013$ & $0.31 \pm 0.02$ \\
\hline IUTD(T6)-fusion & $0.019 \pm 0.006$ & $0.31 \pm 0.02$ \\
\hline
\end{tabular}

The effective molarities (EM) were determined from the ratio of the rate of binding of the OBS1 segment in the fusion construct to OmpF to the rate constant for the bimolecular binding of the $\mathrm{OBS}_{12-18}$ peptide. $\mathrm{EM}_{1}$ and $\mathrm{EM}_{2}$ are effective molarities for the initial binding of the $\mathrm{OBS}_{1}$ segment to $\operatorname{OmpF}\left(k_{12}\right)$ and for the re-binding $\left(k_{12}{ }^{\prime}\right)$, respectively. Rate constants extrapolated to o $\mathrm{mV}$ were used. 